\title{
Focused Ion Beam Fabrication of Solidified Ferritin into Nanoscale Volumes for Compositional Analysis using Time-of-flight Mass Spectrometry Methods
}

\author{
Mark E. Greene, Ty J. Prosa, David J. Larson, and Thomas F. Kelly \\ Imago Scientific Instruments Corporation, 5500 Nobel Dr, Madison, WI 53711
}

We introduce a method to fabricate biological specimens by focused ion beam (FIB) for analysis with atom probe tomography (APT) to assess whether this approach can be used to effectively characterize biomaterials in general [1]. The fabrication methodology is demonstrated using the iron storage protein ferritin composed of a polypeptide shell surrounding an iron-rich nanoparticle core which provides a convenient compositional label for mass spectral analysis [2]. Soft matter is particularly sensitive to ion beam exposure which can induce physical and chemical changes, so any methods developed must include safeguards to minimize specimen damage. We address those issues with protective metal films and low-energy ion fluence to mitigate potential FIB-related problems.

This study had two major objectives: (1) to qualitatively assess the viability of the specimens when subjected to the unique physical conditions of APT analysis, namely ultrahigh vacuum, high electric field, and laser pulsing, and (2) to quantitatively assess the data as a function of various experimental parameters and compare the results with appropriate control specimens. For the first objective, a range of experimental parameters was determined that met the basic criteria necessary to validate that ferritin-based specimens prepared with FIB can be effectively analyzed with APT. Our initial field evaporation time-of-flight mass spectrometry results from APT experiments on Imago LEAP instruments show that the fabricated specimens are capable of emitting ions under various laser pulsing conditions with a high electric field applied. Laser pulse energies on the order of 0.1-1.0 nJ yielded data that suffered from excessive unsynchronized thermal evaporation, while pulse energies $\leq 10 \mathrm{pJ}$ yielded data with significantly lower background and unsynchronized thermal evaporation. Pulse energies on the order of 1-10 pJ and base temperatures $\leq 20 \mathrm{~K}$ were therefore considered optimal APT analysis conditions at a pulse repetition rate of $100 \mathrm{kHz}$.

For the second objective, the experimental parameter space was narrowed to a range that yielded data quality that allowed meaningful spectral comparison between the ferritin-based specimens and the salt-only controls. Comparison revealed that ions originating from the ferritin protein are being detected by APT. Both the ferritin and control spectra contain peaks at $23 \mathrm{Da}$, representing $\mathrm{Na}^{+}$ ions, and a cluster of peaks at 70,72, and 74, representing $\mathrm{Cl}_{2}{ }^{+}$ions. This is consistent with the solidified ferritin material which is expected to contain a great deal of $\mathrm{NaCl}$. The numerous remaining peaks in the ferritin spectrum, however, are attributable to either organic fragments from the amino acid chains or the iron-based core. A significant fraction of the counts at 27 and $28 \mathrm{Da}$ are likely due to $\mathrm{Fe}^{2+}$ ions. After background subtraction, the 28:27 $\mathrm{Da}$ ratio for $\mathrm{Fe}^{2+}$ was found to be approximately 7.0 instead of the ideal value of 15.7 due to convolution with organic species. Going forward, the 27 Da peak can be deconvolved by calibrating the 27:29 Da ratio using apoferritin. Future efforts should provide improved performance on this biomaterial as well as demonstrate expanded results on a broad class of biological materials where 3-D nanoscale compositional mapping is desirable as stand-alone data or as complementary to electron tomography.

References

[1] K. Thompson et al., Ultramicroscopy 107 (2007) 131. 
[2] J. A. Panitz et al., Ultramicroscopy 6 (1981) 3.

[3] We graciously acknowledge John Panitz for thoughtful discussions. This work was supported by DARPA grant W911NF-08-1-0132 through the Army Research Office.
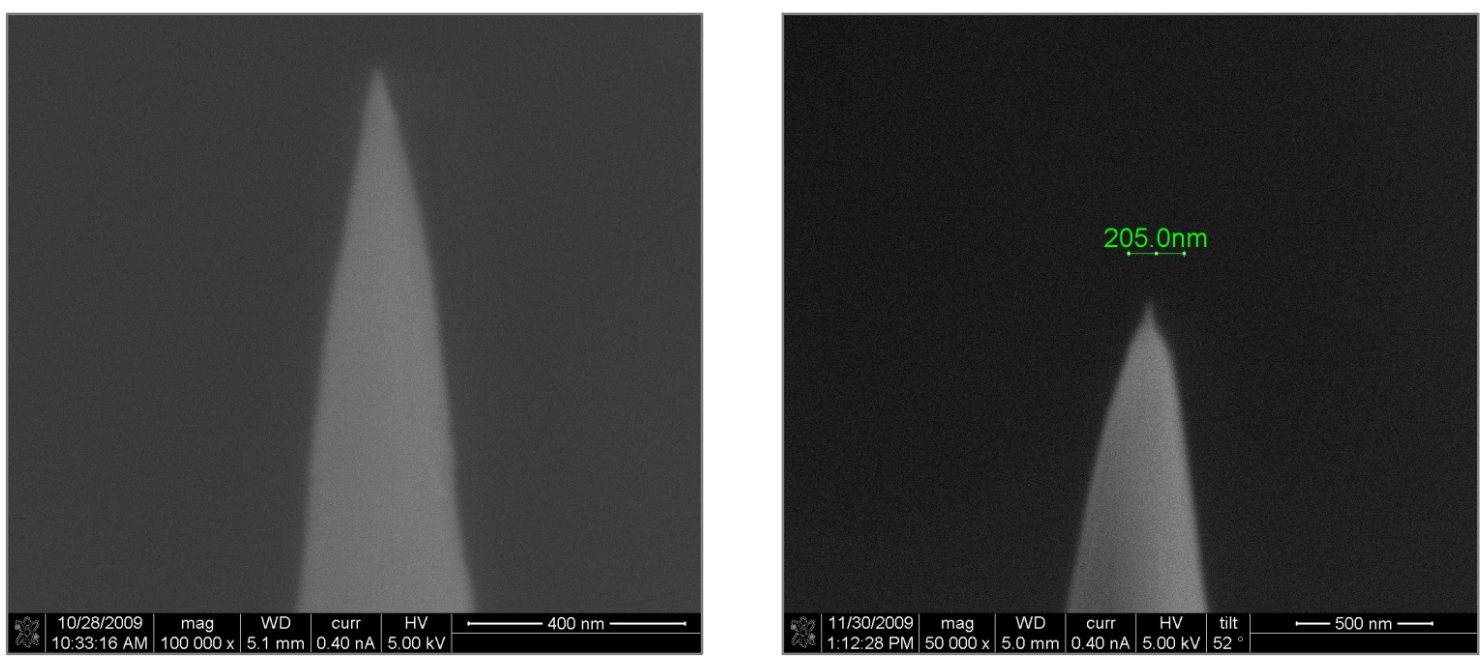

FIG. 1. A solidified ferritin (left) and a salt control (right) specimen prepared by FIB.
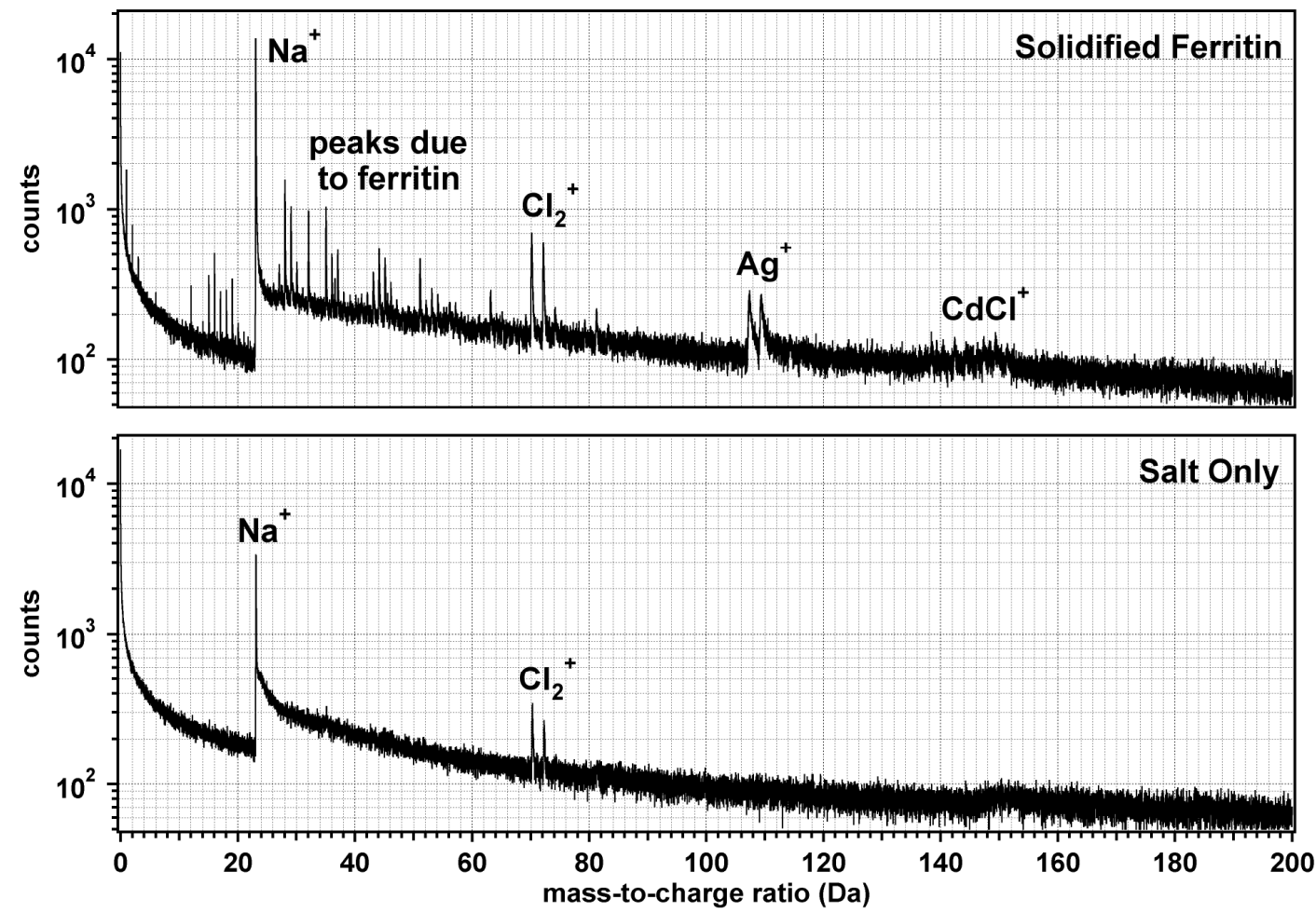

FIG. 2. Mass spectra from a ferritin (top) and a salt control (bottom) specimen collected at laser pulse rate of $100 \mathrm{kHz}$ at a base temperature of $20 \mathrm{~K}$. The ferritin spectrum was collected using $7 \mathrm{pJ}$ laser pulses and the salt control specimen using 5 pJ. Peaks at $23 \mathrm{Da}^{2}$ represent $\mathrm{Na}^{+}$ions and peaks at 70, 72, and 74 represent $\mathrm{Cl}_{2}{ }^{+}$ions. In the ferritin specimen, additional peaks attributable to the protein are evident as are two peaks due to $\mathrm{Ag}^{+}$ions from the protective metal film layer deposited on top of the ferritin material. Mass bins are 0.01 Da. 